\title{
Safety and feasibility of single-incision radical vulvectomy: a novel approach for the treatment of vulvar cancer
}

\author{
Liqing He ${ }^{1 \#} \wedge$, Gaowen Chen ${ }^{1 \#}$, Xiaoxuan $\mathrm{Li}^{1}$, Youhong Zheng ${ }^{1}$, Mengting $\mathrm{Wu}^{1}$, Huiyan Wang ${ }^{1}$, \\ Xiaohong Liu ${ }^{2}$, Wuqi He ${ }^{3}$, Xiaodan Liu ${ }^{4}$, Shaozhuo Huang ${ }^{4}$, Fan Lin ${ }^{4}$, Weixin Liao ${ }^{4}$, Ying Ma ${ }^{1}$, Yifeng Wang ${ }^{1 \wedge}$ \\ ${ }^{1}$ Department of Gynecology, Obstetrics and Gynecology Center, Zhujiang Hospital, Southern Medical University, Guangzhou, China; ${ }^{2}$ Department \\ of Obstetrics and Gynecology, The Fifth People's Hospital of Zhuhai, Zhuhai, China; ${ }^{3}$ Department of Obstetrics and Gynecology, Guilin Women \\ and Children's Medical Center, Guilin, China; ${ }^{4}$ The Second Clinical Medical College, Southern Medical University, Guangzhou, China \\ Contributions: (I) Conception and design: Y Wang, Y Ma, G Chen; (II) Administrative support: None; (III) Provision of study materials or patients: \\ X Li, Y Zheng, H Wang; (IV) Collection and assembly of data: L He, X Li, Y Zheng, M Wu; (V) Data analysis and interpretation: L He, X Li; (VI) \\ Manuscript writing: All authors; (VII) Final approval of manuscript: All authors. \\ "These authors contributed equally to this work. \\ Correspondence to: Yifeng Wang; Ying Ma. Department of Gynecology, Obstetrics and Gynecology Center, Zhujiang Hospital, Southern Medical \\ University, Guangzhou 510280, China. Email: wyf2015@163.com; mayingwuzhuoyi@126.com.
}

Background: In the process of decreasing the morbidity of wound-related complications after vulvectomy and IL for treating vulvar malignancy, we performed a novel surgical procedure-single-incision radical vulvectomy (SIRV). Here, we share our initial experience and report its safety and feasibility.

Methods: Patients with advanced local vulvar tumors were sequentially enrolled in this prospective cohort study to undergo SIRV. While performing SIRV, routine radical vulvectomies were performed first. Subsequently, the flaps of the bridge area between the vulvectomy incisions and femoral triangles were separated and the lymph nodes underneath were removed. Anterior working spaces (AWS) before the femoral triangle were then made. The saphenous vein was carefully identified and retained, while the superficial and deep inguinal lymph nodes were removed from the medial to the lateral sides. After careful hemostasis, the wounds were sutured. Patient demographics, clinical data, pathologic data, operation time, node count, and complications were recorded.

Results: Ten patients underwent SIRV for vulvar cancer. Average hospital stay was $11.70 \pm 3.16$ (range, 9-13) days. The average number of harvested lymph nodes was 7.59 \pm 3.62 (range, 3-15) and 15.14 3.63 (range, 11-20) for per side or both sides of the groin. Blood loss was $\leq 35 \mathrm{~mL}$. Three patients developed inguinal lymphoceles and underwent needle aspirations. Two patients had impaired wound healing and achieved healing after dressing change. No other postoperative complications were noted during follow-up. Conclusions: Compared with conventional open inguinal lymphadenectomy (COIL) and video endoscopic inguinal lymphadenectomy (VEIL), SIRV is a more minimally invasive procedure. Our shortterm observations showed that SIRV is safe and feasible and has good future application prospects for vulvar cancer. However, definitive conclusions cannot be made. Therefore, long-term oncologic outcomes and large-scale clinical trials are warranted.

Keywords: Lymph node excision; minimally invasive surgical procedures; vulvar neoplasms

Submitted Aug 26, 2020. Accepted for publication Nov 26, 2020.

doi: $10.21037 /$ atm-20-6077

View this article at: http://dx.doi.org/10.21037/atm-20-6077

^ ORCID: Liqing He, 0000-0003-3378-9354; Yifeng Wang, 0000-0002-9559-7211. 


\section{Introduction}

Vulvar cancer is the fourth most common gynecologic malignancy, accounting for $5-6 \%$ of female genital tract malignancies. Approximately 6,120 new cases of malignant vulvar tumors are diagnosed each year, and more than 1,350 patients die from vulvar cancer each year in the United States alone (1). With the rising incidence of human papillomavirus (HPV) infection and an aging population, vulvar cancer incidence has increased annually, which has spiked interest among researchers to improve therapeutic methods against vulvar cancer. Lymph node status is an important indicator of patient prognosis. Patients without positive lymph nodes were reported to have a 5 -year overall survival rate of approximately $85 \%$, which sharply declined to $39 \%$ in patients with positive lymph nodes (2). In patients with stage IB or higher vulvar cancer, the standard treatment is radical vulvectomy and inguinal lymphadenectomy (IL). Conventional open IL (COIL) has a higher rate of postoperative wound complications, such as wound dehiscence, necrosis, and lymphocele, at approximately $70 \%$ (3). The application of the separate groin incisions during inguinal lymph node dissection decreases the major complication rate to $21 \%$ (4). However, bridge recurrence occurs more frequently with separate incision techniques (5-8).

Consequently, a large number of studies were conducted to investigate the reduction of postoperative complications of IL. Among these, video endoscopic IL (VEIL) was confirmed to be feasible, together with sentinel lymph node biopsy (SLNB) $(9,10)$. VEIL can significantly decrease the occurrence of wound infection, wound dehiscence, and lymphedema (10-13). However, the need for professional laparoscopic equipment may restrict the overall usage of VEIL and this technique still lacks large-scale studies with long-term oncological outcomes. Furthermore, various studies have shown that SLNB is associated with fewer postoperative complications $(14,15)$; however, for larger $(\geq 40 \mathrm{~mm})$ or multifocal vulvar lesions, SLNB still has a higher recurrence risk; thus, IL is still indicated $(16,17)$.

In the process of exploring the improvement of IL for vulvar cancer, our center performed a novel surgical approach—single-incision radical vulvectomy (SIRV) which involves IL and vulvectomy using a single incision. This study aimed to report the details of the novel procedure and results, including the number of lymph nodes removed, operative time, and wound complications.
We present the following article in accordance with the STROBE reporting checklist (available at http://dx.doi. org/10.21037/atm-20-6077).

\section{Methods}

\section{Study design}

This study is a pilot study conducted with a single arm. In our study, 10 consecutive patients with advanced vulvar cancer who underwent radical vulvectomy and IL from May 2019 to May 2020 at the department of Obstetrics and Gynecology of Zhujiang Hospital were enrolled. The patients were histologically diagnosed with primary vulvar carcinoma, including vulvar squamous cell carcinoma (VSCC), vulvar malignant melanoma, vulvar basal cell carcinoma, and vulvar adenocarcinoma. Women with stage IB, II, IIIA, or IIIB cancer, as defined by the 2009 International Federation of Gynecology and Obstetrics, were included. Radical vulvectomy and unilateral or bilateral IL were planned. Patients with complications such as severe cardiopulmonary disease, mental disorder, or coagulation dysfunction; those with a prior surgical history of the inguinal region; and those with American Anesthesia Association score $>3$ were excluded.

Of the 10 patients who underwent SIRV, six also underwent carbon nanoparticle SLNB prior to vulvectomy. The other four patients were excluded in total because two of them received chemotherapy prior to surgery and two had their lesions removed by biopsy in other hospitals. Patient characteristics, surgical data, pathologic findings, and postoperative complications were recorded. Eight of the patients were diagnosed with VSCC, and the other two were diagnosed with vulvar melanoma and adenocarcinoma. The ages of patients ranged from 55 to 76 years, with an average age of 64 years. Wound dehiscence was defined as the breakdown of more than $25 \%$ of the incision.

Inguinal lymphocele was defined as the accumulation of serous fluid under the surgical field of the inguinal triangle, requiring needle aspiration or even a surgical intervention.

\section{Ethical statement}

The study was conducted in accordance with the Declaration of Helsinki (as revised in 2013). The study was approved by the Ethics board of Zhujiang Hospital (NO. 2019-JS-041-01) and informed consent was taken from all individual participants. 


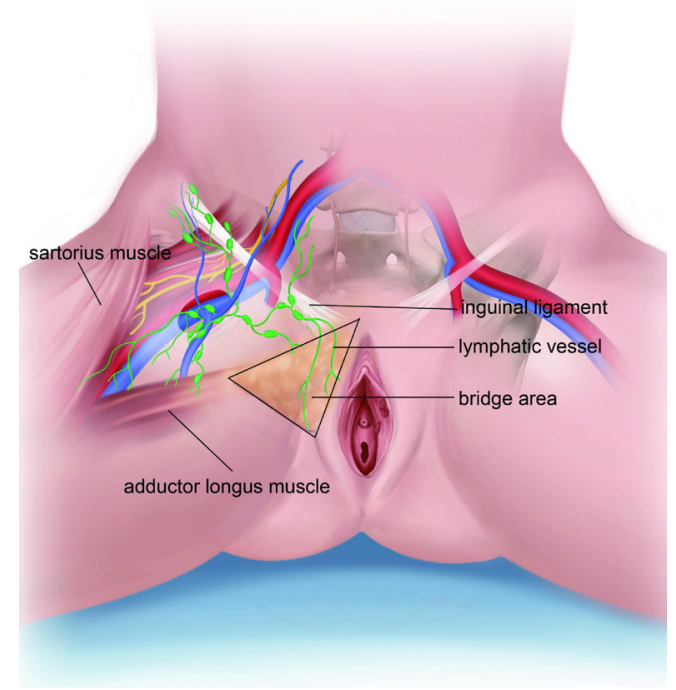

Figure 1 Schematic diagram of bridge area and femoral triangle.

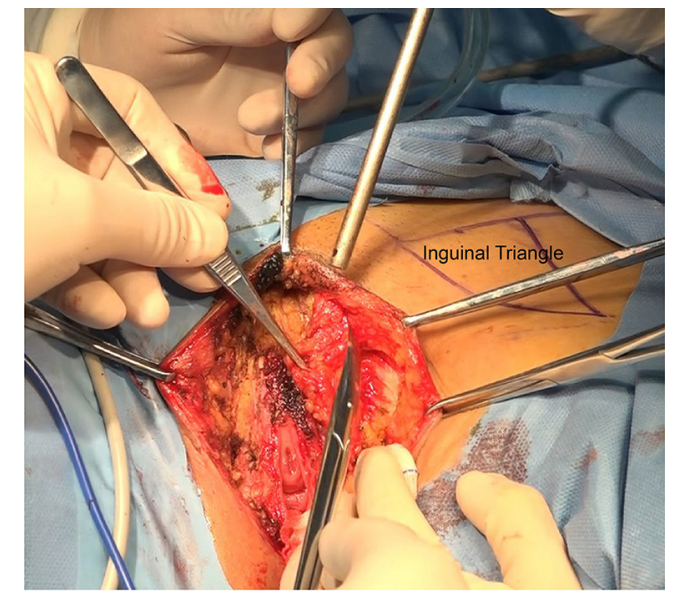

Figure 2 Separation of the lymphoid tissue in bridge area.

\section{Surgical technique}

\section{Patient preparation and position}

Patients were placed in the split-leg position with their legs separated by approximately $80-90^{\circ}$. Partial or complete radical vulvectomy was performed before IL. One milliliter carbon nanoparticle suspension was injected intradermally at four points around the site of the primary tumor after general anesthesia.

\section{Radical vulvectomy}

First, we marked the margin for radical vulvectomy, the "bridge area", and the femoral triangle as indicators
(Figure 1). The surgical margin is $2 \mathrm{~cm}$ in general; however, when a carcinoma closely approaches the urethra or the anus, the tumor-free margin should be $1 \mathrm{~cm}$. The femoral triangle was identified as the region bordered by the inguinal ligament superiorly, sartorius muscle laterally, and adductor longus muscle medially.

A surgical knife was used to cut the vulvar skin layer and dermis along the line, and a short-stalk ultrasonic knife (ETHICON ENDO-SURGERY, LLC Guaynabo, Puerto Rico, US) was used to coagulate and cut the lymphatic adipose tissue of the vulva from the outer ring to the inner ring, with a depth reaching the urogenital fascia. The fascia and tendon convergence on the dorsal side of the clitoris and the perineal complex were directly removed with the ultrasonic knife. A 2-0 absorbable suture using the figure-ofeight method was used to stop the bleeding when needed.

\section{Inguinofemoral lymph node dissection}

IL was then performed through vulvectomy incision.

\section{Forming the anterior working space (AWS)}

Allis tissue forceps were used to elevate the skin and provide traction to the vulvectomy incision edge on the medial side of the pubic tubercle. As a pair of tissue scissors was used to separate the subcutaneous space, aiming to create the initial part of the AWS, including the bridge area (Figure 2). To avoid skin necrosis, this flap included all subcutaneous fat up to the level of the superficial (Camper's) fascia. The Allis tissue forceps were then replaced with a retractor to provide better traction. A harmonic scalpel was used to expand the AWS superiorly, laterally, and inferiorly. The AWS was formed to cover the whole femoral triangle.

\section{Removal of the inguinal nodes}

The superficial lymph nodes lay within the adipose tissue. The adipose tissue was held using long straight tweezers and dissected using a harmonic scalpel. Dissection was performed downward until the medial and lateral edges of the adductor longus were identified. Under direct vision, the inguinal lymph nodes were dissected from the medial edge of the adductor longus muscle to the sartorius muscle (Figure 3). During dissection, the accessory branch of the great saphenous vein, including the superficial external pudendal vessels, superficial epigastric vessels, and superficial circumflex iliac vessels, was encountered and transected individually. Rigorous care was taken to protect the integrity of the great and the accessory great saphenous veins. The nodal bundle was then dissected from the fossa ovalis and the root of the great saphenous vein. Finger palpation was used for the femoral artery 


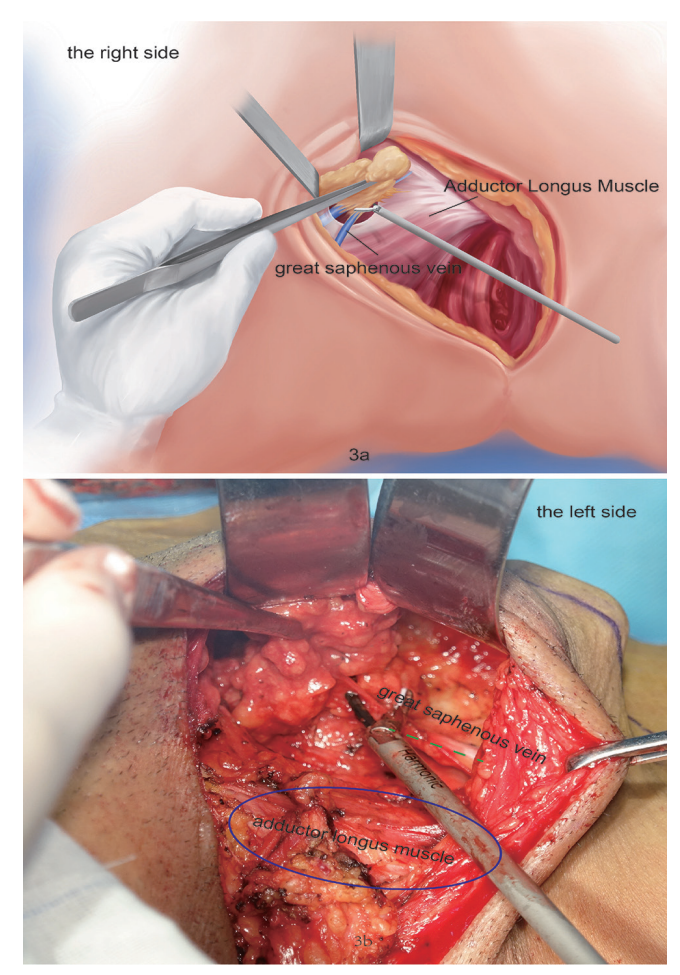

Figure 3 The great saphenous vein was seen during the dissection of superficial inguinal lymph nodes.

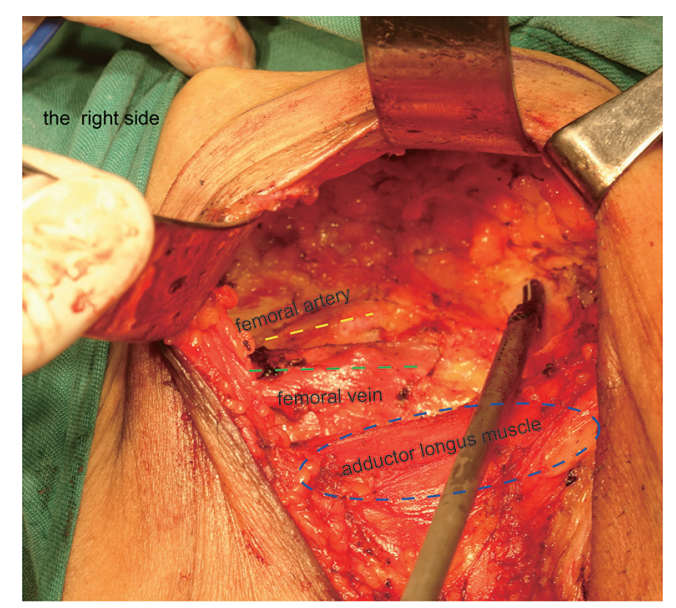

Figure 4 The femoral artery and vein can be seen after the IL.

pulse in a cephalad direction until the junction of the femoral canal, and the inguinal ligament was identified. The deep inguinal lymph nodes were found within the adipose tissue along the anterior and medial surfaces of the femoral vein. The Cloquet node was the uppermost node. After thorough dissection, the femoral vein and artery

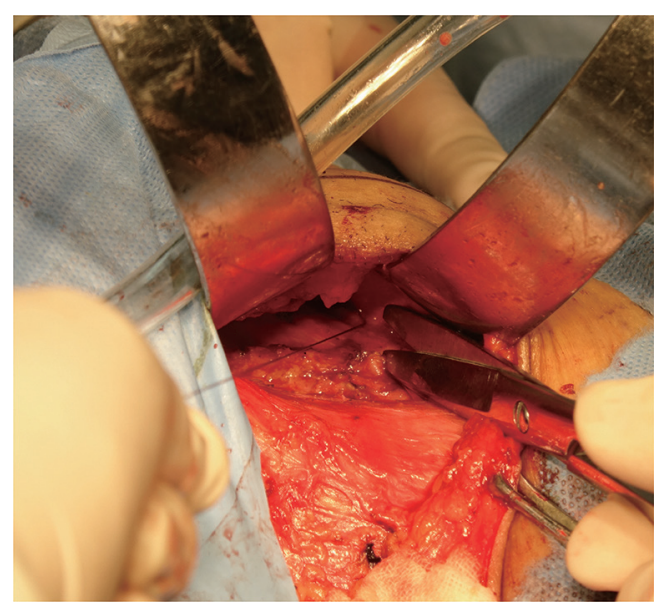

Figure 5 The gap was sutured with 3-0 VICRYL absorbable line.

could be seen (Figure 4). The surgeon could then check the presence of residual lymph nodes by observing and touching the surgical field directly.

\section{Wound closure}

The surgical defect was carefully examined and hemostasis was achieved. In the groin area, the superficial fascia was sutured to the remaining cribriform fascia using a 3-0 VICRYL absorbable suture via the interrupted suture method (Figure 5). No suction drain was placed within the inguinal space (Figure 6).

\section{Postoperative management}

Antibiotics are usually administered postoperatively for 5-7 days. Elastic compression bandage was used to bandage the inguinal region in a "butterfly" shape to reduce lymphatic exudation and promote healing of the surgical field. The patients were kept on bed rest for 3-5 days. A Foley catheter was used for 7 days. To prevent venous thrombosis, intermittent pneumatic compression pump was worn twice a day, and patients were required to perform ankle pump exercise. During this period, the skin color of the lower limbs was observed, and circumference of the thigh, skin temperature, and dorsal artery pulsation were measured to evaluate postoperative complications. Wound dressing changes were performed once a day and suture removal was performed at postoperative day 8 . After taking out the stitches, patients were instructed to have sitz baths with potassium permanganate powder diluted in warm water at a ratio of 1:5,000 once a day for a month. If there were abnormal secretions from the vulva, bacterial culture and drug sensitivity tests were performed and specific antibiotic therapy was initiated. 

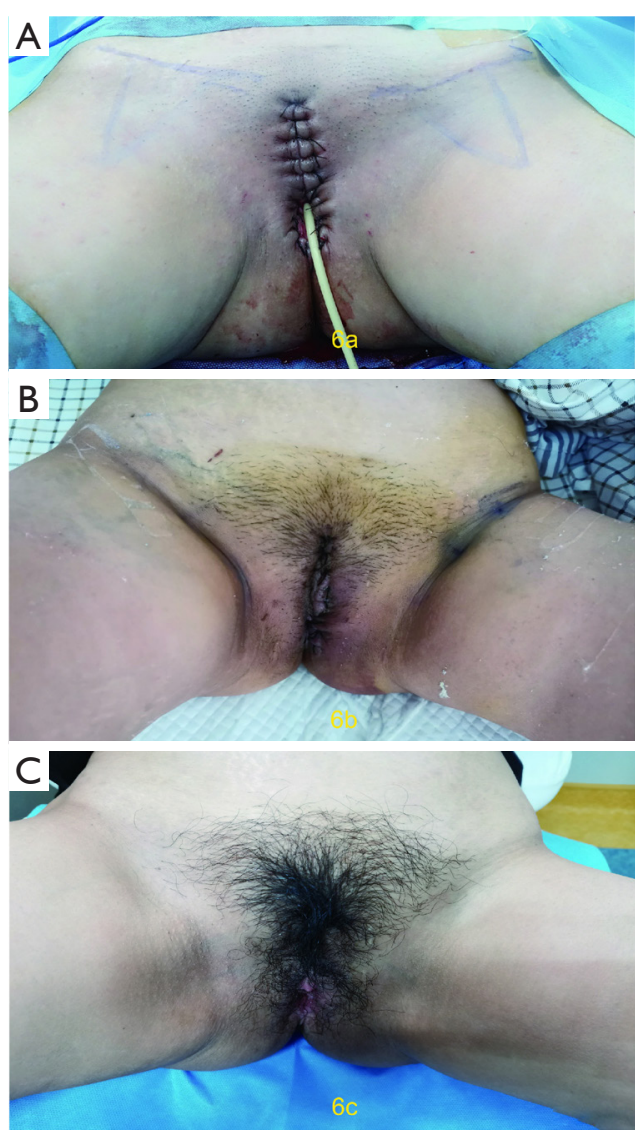

Figure 6 Wound condition at day 0 (A), day 7 (B), and day 90 (C) after the surgery. (A) Wound condition without incisions on the groins. (B) Good wound healing at day 7. (C) The appearance of the vulva and groin seems normal at day 90 .

\section{Follow-up}

Patients were required to undergo a regular outpatient follow-up at least every 3 months for the first 2 years and every 6 months for the later 3-5 years. Gynecological examination, ultrasonography, and tumor marker assessment were mainly performed. Pelvic computed tomography or magnetic resonance imagining was performed when needed.

\section{Statistical analysis}

Descriptive statistics was used in this study. Continuous variables are presented as means with standard deviations and categorical variables as percentages.

\section{Results}

The demographic characteristics of the enrolled patients
Table 1 Demographic characteristics of the ten patients described in this study

\begin{tabular}{ll}
\hline Variable & Result $(\mathrm{n}=10)$ \\
\hline Mean age (year) & $60.20 \pm 12.15$ \\
Range & $41-76$ \\
Mean body mass index $\left(\mathrm{kg} / \mathrm{m}^{2}\right)$ & $23.46 \pm 4.22$ \\
Range & $15.14-29.14$ \\
Histologic type, $\mathrm{n}(\%)$ & \\
VSCC & $8(80.00)$ \\
Melanoma & $1(10.00)$ \\
Adenocarcinoma & $1(10.00)$ \\
FIGO stage, $\mathrm{n}(\%)$ & \\
IB & $6(60.00)$ \\
II & 0 \\
III (IIIA, IIIB, IIIC) & $4(40.00)$ \\
\hline
\end{tabular}

VSCC, vulvar squamous cell carcinoma.

are presented in Table 1. Patients' body mass index ranged from 15.14 to 29.14 (mean, $23.46 \pm 4.22$ ) $\mathrm{kg} / \mathrm{m}^{2}$. Operative and pathologic data from the 10 procedures are presented in Table 2. The operative duration ranged from 80 to 165 (mean, $124.20 \pm 23.29$ ) minutes. Of the 10 patients, seven underwent bilateral IL and the remaining three underwent unilateral IL. Seventeen cases of IL have performed in all. The average number of harvested lymph nodes was $7.59 \pm 3.62$ (range, $3-15$ ) for one side and from $15.14 \pm 3.63$ for both sides. Four patients had positive lymph nodes, with numbers thereof ranging from 1 to 4

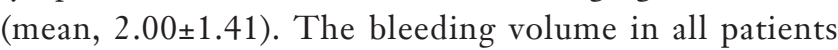
was $\leq 35 \mathrm{~mL}$, and the postoperative hospital stay ranged from 9 to 19 (average, $11.70 \pm 3.16$ ) days. Three patients developed inguinal lymphoceles on postoperative days 8-10 and needle aspirations were performed, followed by elastic compression. Two patients, aged 68 and 72 years, developed poor wound healing. Among them, the more serious patient had a history of diabetes for many years. Her primary lesion was a large tumor located in the lower part of the right labium majus, with a size of $6 \times 5 \times 5 \mathrm{~cm}^{3}$. After resection, the defect was large and vulvar reconstruction was needed. The wound at the flap healed poorly after the operation but improved after debridement. The other patient had poor healing at the posterior perineal junction, which did not require secondary suture and improved after 
Table 2 Operative and pathologic data from the ten procedures performed during this feasibility analysis

\begin{tabular}{ll}
\hline Variable & Result $(\mathrm{n}=10)$ \\
\hline Mean operative time $(\mathrm{min})$ & $124.20 \pm 23.29$ \\
Range & $80.00-165.00$ \\
Mean bleeding volume $(\mathrm{mL})$ & $24.00 \pm 6.15$ \\
Range & $15.00-35.00$ \\
Mean node count (mean) & $7.59 \pm 3.62$ \\
Range & $3-15$ \\
Mean length of stay (days) & $11.70 \pm 3.16$ \\
Range & $9-19$ \\
Postoperative complications & \\
Wound dehiscence & $2 / 10(20.00 \%)$ \\
Inguinal lymphoceles & $3 / 10(30.00 \%)$ \\
Wound infection & 0 \\
\hline
\end{tabular}

dressing change. The remaining eight patients recovered well postoperatively. There were no other long-term complications. No systemic complications, such as deep vein thrombosis, pulmonary embolism, mortality, or cardiac insufficiency, were noted. The surgical margins were negative in all cases.

Six of the 10 patients underwent SLNB. Five patients had black-dyed SLNBs lying in the superficial inguinal lymphatic tissue. Pathological analysis, two patients (33.3\%) had lymph node metastasis.

Inguinal lymph node dissection was subsequently successfully performed in all patients. Based on the pathologic results, four patients with VSCC with positive lymph nodes were recommended to undergo chemotherapy and regional radiation therapy at a total dose of 50-60 Gy. All patients were followed up for at least three months with no cancer recurrence noted in any patients.

\section{Discussion}

The condition of the lymph nodes is an important prognostic factor in vulvar cancer. IL is the standard procedure for locally advanced vulvar cancer, and many advancements have been made to improve it, especially with VEIL and SLNB. Our center first tried to perform IL through the surgical incision in radical vulvectomy as early as May 2019 and achieved good short-term results.
In this study, the mean operative time for SIRV was $124.20 \pm 23.29$ (range, $80-165$ ) minutes, whereas that previously reported $(11,13,18)$ ranged from 45 to 92 minutes, 94 to 170 minutes, and 180 minutes for COIL, VEIL-H, and VEIL-L, respectively. The operative duration required by SIRV is between that required for COIL and VEIL. This is mainly due to the reduction of the scope of the skin incision, leading to a relatively limited operating space. In SIRV, the time-consuming process includes dissection of the lymphoid tissue in the bridge area and closing the lacuna underneath the inguinal region. It is worthwhile to increase the surgical time in exchange for fewer wound complications. Moreover, we speculated that as the surgeon's skills became more proficient with each procedure, the operation time would likely be shortened.

The number of lymph nodes removed during surgery is an important indicator for predicting the prognosis of vulvar cancer. Le (19) and Madeleine Courtney-Brooks (20) have reported that the removal of at least 10 lymph nodes was an independent survival prognostic factor, which was reported to be at least 12 in Baiocchi's study (21). In our study, the average number of harvested lymph nodes during SIRV for bilateral IL was $15.14 \pm 3.63$, which meets the above criterion. In SIRV, the inguinal lymph nodes can be removed under direct vision, and the femoral triangle area can be directly inspected after dissection to eliminate the possibility of remaining enlarged lymph nodes. The average bleeding volume with SIRV reported in this study was $24.00 \pm 6.15$ (range, $15.00-35.00$ ) $\mathrm{mL}$, which is significantly lower than that with COIL $(164.4 \pm 106.5 \mathrm{~mL})$ and VEIL-H $(214.8 \pm 204.4 \mathrm{~mL})$ reported by Zhang et al. (13) and of VEIL-H [137 (range, 80-170) mL] reported by Xu (18). This volume is also slightly higher than the values reported by Le et al. (11). However, we accounted for the blood loss of the total operation, whereas Le accounted for the blood loss of only IL. For postoperative hospital length, the average time at our center was $11.70 \pm 3.16$ days, which was favorable compared to that reported in other centers $(13,19)$.

Regarding postoperative complications, skin necrosis did not occur in any patients, and only one patient needed perineal reconstruction. Compared with COIL, our novel procedure can preserve the integrity of the blood supply of the inguinal skin by making full use of the original incision to perform inguinofemoral lymph node dissection without creating a new wound. Various techniques have been proposed to verify the vitality of vulvar flaps. Magrina (22) and Capozzi (23) evaluated the vitality of the flaps using fluorescein and indocyanine green, respectively. For 
hospitals with certain equipment, this method can be considered to prevent skin necrosis. In our study, three patients developed inguinal lymphocele, which should be due to the large area of lymph-vessel dissection. Fortunately, these were simple lymphoceles without infection, which could be cured by needle aspiration. The large cavity left in the groin area after SIRV is similar with the situation after inguinal lymph node dissection by VEIL. A metaanalysis has shown that lymphatic cyst incidence after VEIL is $0-17.1 \%$ (24). In SIRV, we close this cavity with sutures. We speculate that in the future, lymphatic cyst incidence can be reduced by placing drainage tubes in cases where the skin defect is not too large and can, thus, be directly sutured. For radical resection of larger vulvar lesions, the current trend of vulvar cancer reconstruction is to fill the inguinal dead space after inguinal lymph node dissection, so as to reduce immediate and delayed complications, such as by using Stefano's technique of pedicled anterolateral thigh flap for reconstruction (25).

Although VEIL can significantly reduce surgical complications, its use has not yet been widespread. Jakub et al. $(26,27)$ and Delman (28) have shown that for surgeons to quickly and efficiently master the VEIL technique, it is important for them to have rich experience in COIL surgery, scientific and systematic training, and the guidance of VEIL experts. Specialized laparoscopic equipment is also required. However, it is difficult to meet all these conditions simultaneously. Another important consideration is the lymph node dissection range. A previous study (5) has shown that one of the disadvantages of a separate incision technique is that it may lead to the skin bridge recurrence. One possible mechanism is the existence of tumor emboli in the efferent lymphatic vessels there. Similar concerns may also exist in VEIL, as this technique cannot clean the lymphatic vessels there, either. However, SIRV performed for inguinal lymph node dissection along this pathway can fully clean these efferent lymphatic vessels, which distinguishes it from the separate incision technique and VEIL. When activated carbon was used as a tracer during the operation, black-stained lymphatic vessels could be seen in this area as proof. This raises an important question of whether thorough removal of lymphatic vessels can reduce tumor recurrence and improve survival outcome? This needs to be verified by studies involving large samples and long-term follow-up. In our study, the incidence of lymphedema incidence was low, which may have contributed to the preservation of the great saphenous vein in SIRV This has been demonstrated by Zhang et al. (29) and Dardarian et al. (30) to significantly reduce the incidence of lymphedema complications. Another method to prevent lymphedema is SLNB. Van der Zee et al. (31) have shown that SLNB can significantly reduce shortterm and long-term morbidity including lymphedema of the legs. Many studies have shown that SLNB can decrease postoperative morbidity without compromising the ability to diagnose lymph node metastases $(14,15,17)$. The tracers that have been widely used in the past are dyes and radioisotopes; while the combined effect of dyes and radioisotopes is acceptable, both have their drawbacks. Dyes such as methylene blue diffuse too quickly, easily contaminating of the surgical field and leading to a high rate of false-negatives. Radioisotopes have radioactivity, which is strictly controlled in China. Carbon nanoparticles have a high degree affinity for the lymphatic system and achieved a favorable effect in papillary thyroid microcarcinoma (32) and colorectal cancer (33). In our study, sensitivity was $66.7 \%$, specificity was $100 \%$, and accuracy was $83.3 \%$. Due to the limited sample size, the result was affected by the false-negative cases with a large lesion and positive lymph nodes. Moreover, it is widely accepted that there is a learning curve in performing the SLNB; Morton (34) suggested performing at least 30 consecutive procedures for cutaneous melanoma and de Hullu et al. (35) suggested performing at least 10 procedures for squamous cell carcinoma of the vulva during the learning phase. Largesample studies on the effects of carbon nanoparticles have on vulvar cancer are still warranted. SIRV alone has helped to reduce wound complications; this combined with SLN technology will hopefully further reduce postoperative wound complications.

There are certain limitations to this study that need to be mentioned. First, SIRV is more suitable for large defects at the level of mons pubis and upper part of labia, which would give a more comfortable access to the groin regions. While defects mainly involved the perianal area, this technique is not feasible. Second, when suturing the cavity, an inexperienced surgeon may easily damage large blood vessels. Finally, the number of procedures performed was small, and the follow-up time was too short to evaluate the oncologic outcomes.

\section{Conclusions}

SIRV has a significant advantage over other vulvar cancer radical surgeries in terms of rapid healing and cosmetic effects. The procedure is feasible and safe and has good 
future application prospects. With the number of patients with vulvar cancer increasing yearly, SIRV is an alternative surgical procedure for advanced vulvar malignancy that can reduce medical burden and improve patients' quality of life after surgery. In the future, multi-center, prospective, randomized trials could help to further establish the optimal use of SIRV in the treatment of patients with advanced vulvar malignancy.

\section{Acknowledgments}

Funding: This work was supported by grants from Guangdong Province University Student Innovation and Entrepreneurship Training Program (grant number 201612121058)

\section{Footnote}

Reporting Checklist: The authors have completed the STROBE reporting checklist. Available at http://dx.doi. org/10.21037/atm-20-6077

Data Sharing Statement: Available at http://dx.doi. org/10.21037/atm-20-6077

Conflicts of Interest: All authors have completed the ICMJE uniform disclosure form (available at http://dx.doi. org/10.21037/atm-20-6077). The authors have no conflicts of interest to declare.

Ethical Statement: The authors are accountable for all aspects of the work in ensuring that questions related to the accuracy or integrity of any part of the work are appropriately investigated and resolved. The study was performed in compliance with the ethical principles of the Declaration of Helsinki (as revised in 2013) and had been approved by the Ethical Board of Zhujiang Hospital (NO. 2019-JS-041-01). Informed consent was obtained from all patients before surgery.

Open Access Statement: This is an Open Access article distributed in accordance with the Creative Commons Attribution-NonCommercial-NoDerivs 4.0 International License (CC BY-NC-ND 4.0), which permits the noncommercial replication and distribution of the article with the strict proviso that no changes or edits are made and the original work is properly cited (including links to both the formal publication through the relevant DOI and the license).
See: https://creativecommons.org/licenses/by-nc-nd/4.0/.

\section{References}

1. Siegel RL, Miller KD, Jemal A. Cancer statistics, 2020. CA: A Cancer Journal for Clinicians 2020;70:7-30.

2. Cavanagh D, Fiorica JV, Hoffman MS, et al. Invasive carcinoma of the vulva. Changing trends in surgical management. Am J Obstet Gynecol 1990;163:1007-15.

3. Gatta G, Capocaccia R, Trama A, et al. The burden of rare cancers in Europe. Adv Exp Med Biol 2010;686:285-303.

4. Hacker NF, Leuchter RS, Berek JS, et al. Radical vulvectomy and bilateral inguinal lymphadenectomy through separate groin incisions. Obstet Gynecol 1981;58:574-9.

5. Christopherson W, Buchsbaum HJ, Voet R, et al. Radical vulvectomy and bilateral groin lymphadenectomy utilizing separate groin incisions: report of a case with recurrence in the intervening skin bridge. Gynecol Oncol 1985;21:247-51.

6. Rose PG. Skin bridge recurrences in vulvar cancer: frequency and management. Int J Gynecol Cancer 1999;9:508-11.

7. De Hullu JA, Hollema H, Lolkema S, et al. Vulvar carcinoma. The price of less radical surgery. Cancer 2002;95:2331-8.

8. Rouzier R, Haddad B, Plantier F, et al. Local relapse in patients treated for squamous cell vulvar carcinoma: incidence and prognostic value. Obstet Gynecol 2002;100:1159-67.

9. Johann S, Klaeser B, Krause T, et al. Comparison of outcome and recurrence-free survival after sentinel lymph node biopsy and lymphadenectomy in vulvar cancer. Gynecol Oncol 2008;110:324-8.

10. Wang YF, Chen GW, Weng HN, et al. Surgical technique of video endoscopic inguinal lymphadenectomy via a hypogastric subcutaneous approach. Chin Med J (Engl) 2013;126:3181-3.

11. Le A, Xiong J, Wang Z, et al. Endoscopy-assisted inguinal lymphadenectomy in vulvar cancer. Arch Gynecol Obstet 2018;297:1277-83.

12. Wu Q, Gong Z, Zhao Y, et al. Video Endoscopic Inguinal Lymphadenectomy via 3-Incision Lateral Approach for Vulvar Cancers: Our Preliminary Outcome of 37 Cases. Int J Gynecol Cancer 2016;26:1706-11.

13. Zhang $M$, Chen L, Zhang X, et al. A Comparative Study of Video Endoscopic Inguinal Lymphadenectomy and Conventional Open Inguinal Lymphadenectomy 
for Treating Vulvar Cancer. Int J Gynecol Cancer 2017;27:1983-9.

14. Cham S, Chen L, Burke WM, et al. Utilization and Outcomes of Sentinel Lymph Node Biopsy for Vulvar Cancer. Obstet Gynecol 2016;128:754-60.

15. Rodríguez-Trujillo A, Fusté $\mathrm{P}$, Paredes $\mathrm{P}$, et al. Long-term oncological outcomes of patients with negative sentinel lymph node in vulvar cancer. Comparative study with conventional lymphadenectomy. Acta Obstet Gynecol Scand 2018;97:1427-37.

16. Hinten F, van den Einden LC, Hendriks JC, et al. Risk factors for short- and long-term complications after groin surgery in vulvar cancer. Br J Cancer 2011;105:1279-87.

17. Nica A, Covens A, Vicus D, et al. Sentinel lymph nodes in vulvar cancer: Management dilemmas in patients with positive nodes and larger tumors. Gynecol Oncol 2019;152:94-100.

18. Xu H, Wang D, Wang $\mathrm{Y}$, et al. Endoscopic inguinal lymphadenectomy with a novel abdominal approach to vulvar cancer: description of technique and surgical outcome. J Minim Invasive Gynecol 2011;18:644-50.

19. Le T, Elsugi R, Hopkins L, et al. The definition of optimal inguinal femoral nodal dissection in the management of vulva squamous cell carcinoma. Ann Surg Oncol 2007;14:2128-32.

20. Courtney-Brooks M, Sukumvanich P, Beriwal S, et al. Does the number of nodes removed impact survival in vulvar cancer patients with node-negative disease? Gynecol Oncol 2010;117:308-11.

21. Baiocchi G, Cestari FM, Rocha RM, et al. Does the count after inguinofemoral lymphadenectomy in vulvar cancer correlate with outcome? Eur J Surg Oncol 2013;39:339-43.

22. Magrina JF, Masterson BJ. Evaluation of groin and vulvar flap viability by the use of intravenous fluorescein. Gynecol Oncol 1981;11:96-101.

23. Capozzi VA, Ceni V, Sozzi G, et al. Endoscopic near infrared and indocyanine green to verify the viability of the subcutaneous flap for vulvar cancer. Gynecol Oncol 2019;154:653-4.

24. Liu CE, Lu Y, Yao DS. Feasibility and Safety of Video Endoscopic Inguinal Lymphadenectomy in Vulvar Cancer: A Systematic Review. PLoS One 2015;10:e0140873.

25. Gentileschi S, Servillo M, Garganese G, et al. Versatility of pedicled anterolateral thigh flap in gynecologic reconstruction after vulvar cancer extirpative surgery. Microsurgery 2017;37:516-24.
26. Jakub JW, Terando AM, Sarnaik A, et al. Training High-Volume Melanoma Surgeons to Perform a Novel Minimally Invasive Inguinal Lymphadenectomy: Report of a Prospective Multi-Institutional Trial. J Am Coll Surg 2016;222:253-60.

27. Jakub JW, Terando AM, Sarnaik A, et al. Safety and Feasibility of Minimally Invasive Inguinal Lymph Node Dissection in Patients With Melanoma (SAFE-MILND): Report of a Prospective Multi-institutional Trial. Ann Surg 2017;265:192-6.

28. Delman KA. Minimally invasive inguinal lymphadenectomy. J Surg Oncol 2020;122:96-8.

29. Zhang SH, Sood AK, Sorosky JI, et al. Preservation of the saphenous vein during inguinal lymphadenectomy decreases morbidity in patients with carcinoma of the vulva. Cancer 2000;89:1520-5.

30. Dardarian TS, Gray HJ, Morgan MA, et al. Saphenous vein sparing during inguinal lymphadenectomy to reduce morbidity in patients with vulvar carcinoma. Gynecol Oncol 2006;101:140-2.

31. Van der Zee AG, Oonk MH, De Hullu JA, et al. Sentinel node dissection is safe in the treatment of early-stage vulvar cancer. J Clin Oncol 2008;26:884-9.

32. Hao RT, Chen J, Zhao LH, et al. Sentinel lymph node biopsy using carbon nanoparticles for Chinese patients with papillary thyroid microcarcinoma. Eur J Surg Oncol 2012;38:718-24.

33. Cai HK, He HF, Tian W, et al. Colorectal cancer lymph node staining by activated carbon nanoparticles suspension in vivo or methylene blue in vitro. World J Gastroenterol 2012;18:6148-54.

34. Morton DL, Thompson JF, Essner R, et al. Validation of the accuracy of intraoperative lymphatic mapping and sentinel lymphadenectomy for early-stage melanoma: a multicenter trial. Multicenter Selective Lymphadenectomy Trial Group. Ann Surg 1999;230:453-63; discussion 463-5.

35. de Hullu JA, Hollema H, Piers DA, et al. Sentinel lymph node procedure is highly accurate in squamous cell carcinoma of the vulva. J Clin Oncol 2000;18:2811-6.

Cite this article as: He L, Chen G, Li X, Zheng Y, Wu M, Wang H, Liu X, He W, Liu X, Huang S, Lin F, Liao W, Ma Y, Wang Y. Safety and feasibility of single-incision radical vulvectomy: a novel approach for the treatment of vulvar cancer. Ann Transl Med 2021;9(4):320. doi: 10.21037/atm-20-6077 